\title{
84. Cytogenetic Data of 92 Cases Obtained in the Kojika-Gakuen, an Institution for Severe Physically and Mentally Retarded Patients
}

\author{
By Tetsuji Kadotani, *) Yoko Watanabe,*) Takako Kiyuna,*) \\ Toshimi KAWAmoto,*) and Ichiro TAKemURA**) \\ (Communicated by Sajiro Makino, M. J. A., Sept. 12, 1980)
}

Since 1978, cytogenetic information has been presented in 205 cases of congenital defectives and their relatives derived from genetic counseling at an institution for the congenital defectives.***) Additional data were accumulated in 92 cases from the Kojika-Gakuen, an institution for severe physically and mentally retarded patients. The present paper describes chromosomal findings in those cases in the form of summary together with their clinical features. This is a product of the combination-work of the Kojika-Gakuen and the Kadotani Medical Research Foundation.

According to the standard blood culture technique, chromosomal studies were carried out in $\mathbf{9 2}$ defectives and their relatives (for detail, refer to Table I). In each case, chromosome counts were made in 50 or more cells, and at least 10 cells with the modal chromosomenumber were karyotyped. In cases with abnormal karyotypes, over 10 cells were analysed following the G- and Q-banding procedures.

Results and remarks. Table I shows the results of clinical examinations and chromosomal observations in 92 cases so far studied. Seventy among them were found to possess a normal karyotype, leaving 22 cases showing abnormal karyotypes, being $24 \%$ in incidence. They were represented by one case of triple $X$; two cases of the long $\mathrm{Y}$; four cases of the short $\mathrm{Y}$; two cases of the elongated short arm of no. 22; one case of the elongated satellite of no. 21 ; one case of the deleted short arm of no. 21; one case of trisomy 21 ; one case of the elongated short arm of D chromosome; one case of the elongated short arm of no. 15; one case of elongated satellite of no. 14 ; three cases of the elongated short arm of no. 13; one case of a mosaicism with ring no. $9(46, \mathrm{XX} / 46, \mathrm{XX}, \mathrm{r}(9)(\mathrm{p} 24 \mathrm{q} 34))$; one case of the ab-

*) The Kadotani Medical Research Foundation. Saijohigashi, Higashihiroshima, Hiroshima.

**) Kojika-Gakuen. Awayacho, Miyoshi, Hiroshima.

***) Katano et al.: Proc. Japan Acad., vol. 54B, pp. 111-115, 1978; vol. 56B, pp. 263-267, 1980. 
Table I. Clinical notes and chromosomal findings of 92 cases from the KojikaGakuen for mentally retarded and physically defected patients

\begin{tabular}{|c|c|c|c|c|}
\hline $\begin{array}{l}\text { Case } \\
\text { no. }\end{array}$ & Age & Sex & Karyotype & Clinical feature \\
\hline 1 & $6 \mathrm{y}$. & $\mathbf{F}$ & $46, \mathrm{XX}$ & $\begin{array}{l}\text { slow growth, malnutrition, flat occiput, strabismus, shield } \\
\text { chest, palsy of extremity, ataxia, idiot }\end{array}$ \\
\hline $2 *$ & $9 y$. & $\mathrm{F}$ & $46, \mathrm{XX}, 13 \mathrm{p}+$ & $\begin{array}{l}\text { slow growth, malnutrition, microcephaly, ataxia, disturb- } \\
\text { ance of gaits, idiot }\end{array}$ \\
\hline $3 *$ & $38 \mathrm{y}$. & M & $46, X Y, 13 p+$ & father of the case no. 2 \\
\hline 4 & $34 \mathrm{y}$ & $F$ & $46, \mathrm{XX}$ & mother of the case no. 2 \\
\hline $5^{*}$ & $3 \mathrm{y}$. & M & $46, X Y, 13 p+$ & younger brother of the case no. 2 \\
\hline 6 & $10 \mathrm{y}$. & $\mathrm{F}$ & $46, \mathrm{XX}$ & $\begin{array}{l}\text { slow growth, malnutrition, flat occiput, round face, hyper- } \\
\text { telorism, upward slanted palpebral fissure, dislocation of } \\
\text { hip, recurrent cramp, ataxia, idiot }\end{array}$ \\
\hline $7 \%$ & $7 \mathrm{y}$. & $\mathrm{F}$ & $46, \mathrm{XX}, \mathrm{Dp}+$ & $\begin{array}{l}\text { slow growth, malnutrition, strabismus, atrial septal de- } \\
\text { fect, palsy of extremity, hypotonicity, ataxia, idiot }\end{array}$ \\
\hline 8 & $8 \mathrm{y}$. & M & $46, \mathrm{XY}$ & $\begin{array}{l}\text { low birth weight, spina bifida, clubfoot, valgus, palsy of } \\
\text { extremity, athetotic movement, hypertonicity, recurrent } \\
\text { cramp, ataxia, dysbasis, idiot }\end{array}$ \\
\hline 9 & $31 \mathrm{y}$. & $\mathrm{F}$ & $46, \mathrm{XX}$ & $\begin{array}{l}\text { slow growth, malnutrition, round face, mask-like facies, } \\
\text { palsy of extremity, hypertonicity, ataxia, disturbance of } \\
\text { gaits, idiot }\end{array}$ \\
\hline 10 & $9 \mathrm{y}$. & M & $46, \mathrm{XY}$ & $\begin{array}{l}\text { slow growth, malnutrition, short neck, round face, weak } \\
\text { sight, palsy of extremity, muscular atrophy, ataxia, dis- } \\
\text { turbance of gaits, idiot }\end{array}$ \\
\hline $11^{*}$ & $29 \mathrm{y}$. & $\mathrm{F}$ & $46, \mathrm{XXX}$ & $\begin{array}{l}\text { slow growth, malnutrition, short neck, round face, cleft lip, } \\
\text { teeth anomaly and irregular placement, atresia ani and } \\
\text { vulvae, rocker bottom, palsy of extremity, ataxia, disturb- } \\
\text { ance of gaits, idiot }\end{array}$ \\
\hline 12 & $14 \mathrm{y}$. & M & $46, \mathrm{XY}$ & $\begin{array}{l}\text { flat occiput, strabismus, incomplete development of exter- } \\
\text { nal genitalia, small penis, palsy of extremity, hypertonici- } \\
\text { ty, ataxia, disturbance of gaits, idiot }\end{array}$ \\
\hline $13^{*}$ & $17 \mathrm{y}$. & M & $46, X Y, 22 p+$ & $\begin{array}{l}\text { slow growth, clubhand, clubfoot, palsy of extremity, hyper- } \\
\text { tonicity, recurrent cramp, ataxia, dysbasia, idiot }\end{array}$ \\
\hline $14^{*}$ & $48 \mathrm{y}$. & $\mathrm{F}$ & $46, X X, 22 p+$ & mother of the case no. 13 \\
\hline 15 & $11 \mathrm{y}$. & $\mathrm{F}$ & $46, \mathrm{XX}$ & younger sister of the case no. 13 \\
\hline $16^{*}$ & $12 \mathrm{y}$. & $\mathrm{F}$ & $46, \mathrm{XX}, \operatorname{dir} \mathrm{du}$ & $\begin{array}{l}\text { pp(3) }(\mathrm{q} 22 \rightarrow \mathrm{q} 27) \\
\text { low birth weight, sparse scalp hair, flat occiput, mask-like } \\
\text { facies, recurrent cramp, ataxia, disturbance of gaits, idiot }\end{array}$ \\
\hline 17 & $34 \mathrm{y}$. & M & $46, \mathrm{XY}$ & father of the case no. 16 \\
\hline 18 & $36 \mathrm{y}$. & $\mathrm{F}$ & $46, \mathrm{XX}$ & mother of the case no. 16 \\
\hline 19 & $8 \mathrm{y}$ & $\mathrm{F}$ & $46, \mathrm{XX}$ & $\begin{array}{l}\text { slow growth, malnutrition, low birth weight, flat occiput, } \\
\text { weak sight, palsy of extremity, muscular atrophy, ataxia, } \\
\text { disturbance of gaits, idiot }\end{array}$ \\
\hline 20 & $5 \mathrm{y}$. & $\mathrm{F}$ & $46, \mathrm{XX}$ & $\begin{array}{l}\text { slow growth, malnutrition, low birth weight, nystagmus, } \\
\text { pigeon breast, low-leg, palsy of extremity, hypertonicity, } \\
\text { recurrent cramp, ataxia, disturbance of gaits, idiot }\end{array}$ \\
\hline 21 & $8 \mathrm{y}$ & M & $46, \mathrm{XY}$ & $\begin{array}{l}\text { slow growth, malnutrition, short neck, round face, upward } \\
\text { slanted palpebral fissure, teeth anomaly and irregular } \\
\text { placement, clubhand, clubfoot, hypertonicity, hyperreflex } \\
\text { of the tendon, recurrent cramp, ataxia, dysbasia, idiot }\end{array}$ \\
\hline 22 & $10 \mathrm{y}$. & M & $46, \mathrm{XY}$ & $\begin{array}{l}\text { dolichocephalia, palsy of extremity, ataxia, disturbance of } \\
\text { gaits, imbecile }\end{array}$ \\
\hline 23 & $34 \mathrm{y}$. & $\mathrm{F}$ & $46, \mathrm{XX}$ & $\begin{array}{l}\text { slow growth, malnutrition, flat occiput, degeneration of } \\
\text { retinal pigment, weak sight, saddle nose, low voice, teeth } \\
\text { anomaly and irregular placement, palsy of extremity, } \\
\text { muscular atrophy, recurrent cramp, ataxia, disturbance of } \\
\text { gaits, debility }\end{array}$ \\
\hline 24 & $9 \mathrm{y}$. & M & $46, X Y$ & $\begin{array}{l}\text { slow grorvth, malnutrition, hydrocephaly, frontal bossing, } \\
\text { teeth anomaly and irregular placement, short neck, club- } \\
\text { foot, clubhand, incomplete development of internal and } \\
\text { external genitalia, palsy of extremity, hypertonicity, re- } \\
\text { current cramp, ataxia, disturbance of gaits, idiot }\end{array}$ \\
\hline 25 & $6 \mathrm{y}$. & M & $46, \mathrm{XY}$ & $\begin{array}{l}\text { slow growth, malnutrition, flat occiput, clubfoot, equinus, } \\
\text { recurrent cramp, ataxia, disturbance of gaits, idiot }\end{array}$ \\
\hline 26 & $6 \mathrm{y}$. & $\mathrm{F}$ & $46, \mathrm{XX}$ & $\begin{array}{l}\text { slow growth, malnutrition, flat occiput, palsy of extremity, } \\
\text { hypertonicity, recurrent cramp, ataxia, disturbance of } \\
\text { gaits, idiot }\end{array}$ \\
\hline 27 & $9 \mathrm{y}$. & $F^{4}$ & $46, \mathrm{XX}$ & $\begin{array}{l}\text { flat occiput, weak sight, palsy of extremity, ataxiä, dis- } \\
\text { turbance of gaits, idiot }\end{array}$ \\
\hline
\end{tabular}


Table I (Continued)

\begin{tabular}{|c|c|c|c|c|}
\hline $\begin{array}{l}\text { Case } \\
\text { no. }\end{array}$ & Age & Sex & Karyotype & Clinical feature \\
\hline 28 & $25 \mathrm{y}$. & $\mathrm{M}$ & $46, \mathrm{XY}$ & $\begin{array}{l}\text { saddle nose, low voice, clubfoot, equinus, rocker bottom, } \\
\text { palsy of extremity, hypertonicity, malformed auricle } \\
\text { (right), ataxia, disturbance of gaits, debility }\end{array}$ \\
\hline 29 & $7 \mathrm{y}$. & M & $46, X Y$ & $\begin{array}{l}\text { microcephaly, oxycephaly, flat occiput, ataxia, disturbance } \\
\text { of gaits, idiot }\end{array}$ \\
\hline 30 & $20 \mathrm{y}$. & $\mathrm{M}$ & $46, \mathrm{XY}$ & $\begin{array}{l}\text { flat occiput teeth anomaly and irregular placement, club- } \\
\text { hand, clubfoot, calcaneus, rocker bottom, palsy of ex- } \\
\text { tremity, muscle atrophy, recurrent cramp, ataxia, dis- } \\
\text { turbance of gaits, idiot }\end{array}$ \\
\hline 31 & $24 \mathrm{y}$. & M & $46, \mathrm{XY}$ & $\begin{array}{l}\text { macroglossia, clubfoot, equinovarus, dislocation of hip, } \\
\text { palsy of extremity, ataxia, disturbance of gaits, idiot }\end{array}$ \\
\hline 32 & $11 \mathrm{y}$. & $\mathrm{F}$ & $46, \mathrm{XX}$ & $\begin{array}{l}\text { flat occiput, strabismus, palsy of extremity, muscular atro- } \\
\text { phy, ataxia, disturbance of gaits, idiot }\end{array}$ \\
\hline 33 & $16 \mathrm{y}$. & M & $46, \mathrm{XY}$ & $\begin{array}{l}\text { sparse scalp hair, dolichocephalia, flat occiput, frontal } \\
\text { bossing, upward slanted palpebral fissure, exophthalmus, } \\
\text { nystagmus, weak sight, palsy of extremity, muscular atro- } \\
\text { phy, ataxia, disturbance of gaits, idiot }\end{array}$ \\
\hline 34 & $49 \mathrm{y}$ & M & $46, \mathrm{XY}$ & $\begin{array}{l}\text { microcephaly, cataract, weak sight, dysplasia of right I. } \\
\text { finger, ataxia, disturbance of gaits, imbecile }\end{array}$ \\
\hline $35^{*}$ & 15 у. & $\mathrm{M}$ & $46, X Y q-$ & $\begin{array}{l}\text { clubhand, clubfoot, equinus, palsy of extremity, hyper- } \\
\text { tonicity, ataxia, disturbance of gaits }\end{array}$ \\
\hline 36 & $21 \mathrm{y}$. & $\mathrm{M}$ & $46, X Y$ & $\begin{array}{l}\text { sparse scalp hair, microcephaly, flat occiput, short neck, } \\
\text { protrusion of labium inferius, broad finger and toe, club- } \\
\text { hand, clubfoot, equinus, rocker bottom, palsy of extremity, } \\
\text { recurrent cramp, ataxia, disturbance of gaits, idiot }\end{array}$ \\
\hline 37 & $10 \mathrm{y}$. & M & $46, X Y$ & $\begin{array}{l}\text { slow growth, flat occiput, clubfoot, palsy of extremity, } \\
\text { ataxia, disturbance of gaits, idiot }\end{array}$ \\
\hline $38 *$ & $14 \mathrm{y}$. & M & $46, \mathrm{XYq}+$ & $\begin{array}{l}\text { short stature, microcephaly, clubfoot, equinus, palsy of } \\
\text { extremity, ataxia, disturbance of gaits, idiot }\end{array}$ \\
\hline 39 & $8 \mathrm{y}$. & $\mathrm{F}$ & $46, \mathrm{XX}$ & $\begin{array}{l}\text { clubfoot, equinus, palsy of extremity, recurrent cramp, } \\
\text { ataxia, disturbance of gaits, idiot }\end{array}$ \\
\hline 40 & $19 \mathrm{y}$. & $\mathrm{F}$ & $46, \mathrm{XX}$ & $\begin{array}{l}\text { clubfoot, equinovarus, palsy of extremity, hypertonicity, } \\
\text { ataxia, disturbance of gaits }\end{array}$ \\
\hline 41 & $48 \mathrm{y}$. & $\mathrm{F}$ & $46, \mathrm{XX}$ & $\begin{array}{l}\text { aged face, weak sight, equinus, rocker bottom, palsy of } \\
\text { extremity, recurrent cramp, ataxia, disturbance of gaits, } \\
\text { idiot }\end{array}$ \\
\hline 42 & $28 \mathrm{y}$. & F & $46, \mathrm{XX}$ & $\begin{array}{l}\text { microcephaly, flat occiput, weak sight, teeth anomaly and } \\
\text { irregular placement, clubfoot, palsy of extremity, muscular } \\
\text { atrophy, ataxia, disturbance of gaits, idiot }\end{array}$ \\
\hline 43 & $21 \mathrm{y}$. & $\mathrm{F}$ & $46, \mathrm{XX}$ & $\begin{array}{l}\text { flat occiput, flat broad facies, upward slanted palpebral } \\
\text { fissure, low nasal bridge, palsy of extremity, recurrent } \\
\text { cramp, ataxia, disturbance of gaits, idiot }\end{array}$ \\
\hline 44 & $16 \mathrm{y}$. & $\mathrm{F}$ & $46, \mathrm{XX}$ & $\begin{array}{l}\text { saddle nose, palsy of extremity, ataxia, disturbance of } \\
\text { gaits, idiot }\end{array}$ \\
\hline 45 & $14 \mathrm{y}$. & $\mathrm{F}$ & $46, \mathrm{XX}$ & $\begin{array}{l}\text { exophthalmus, cyanosis of extremity, palsy of extremity, } \\
\text { ataxia, disturbance of gaits, idiot }\end{array}$ \\
\hline 46 & $18 \mathrm{y}$. & $\mathrm{F}$ & $46, \mathrm{XX}$ & $\begin{array}{l}\text { strabism, saddle nose, cyanosis of extremity, palsy of ex- } \\
\text { tremity, recurrent cramp, ataxia, disturbance of gaits, } \\
\text { idiot }\end{array}$ \\
\hline 47 & $38 \mathrm{y}$. & M & $46, \mathrm{XY}$ & $\begin{array}{l}\text { rocker bottom, palsy of extremity, ataxia, disturbance of } \\
\text { gaits, imbecile }\end{array}$ \\
\hline 48 & $16 \mathrm{y}$. & $\mathrm{F}$ & $46, \mathrm{XX}$ & $\begin{array}{l}\text { slow growth, malnutrition, dry skin, microcephaly, stra- } \\
\text { bism, saddle nose, low nasal bridge, protrusion of labium } \\
\text { inferius, incomplete development of internal and external } \\
\text { genitalia, wide cleft between I. and II. toe }\end{array}$ \\
\hline 49 & $12 \mathrm{y}$. & M & $46, \mathrm{XY}$ & $\begin{array}{l}\text { slow growth, malnutrition, short stature, short neck, in- } \\
\text { complete development of external genitalia, rocker bot- } \\
\text { tom, palsy of extremity, hypertonicity, ataxia, disturbance } \\
\text { of gaits, imbecile }\end{array}$ \\
\hline 50 & $23 \mathrm{y}$ & $\mathrm{F}$ & $46, \mathrm{XX}$ & $\begin{array}{l}\text { dry skin, short neck, round face, facial telangiectatic ery- } \\
\text { thema, flat occiput, microphthalmus, blindness, saddle } \\
\text { nose, teeth anomaly and irregular placement, rocker bot- } \\
\text { tom, recurrent cramp, ataxia, idiot }\end{array}$ \\
\hline 51 & $15 \mathrm{y}$. & $\mathrm{F}$ & $46, \mathrm{XX}$ & $\begin{array}{l}\text { malnutrition, clubfoot, equinus, palsy of extremity, hyper- } \\
\text { tonicity, recurrent cramp, ataxia, disturbance of gaits, } \\
\text { debility }\end{array}$ \\
\hline
\end{tabular}


Table I (Continued)

\begin{tabular}{|c|c|c|c|c|}
\hline $\begin{array}{l}\text { Case } \\
\text { no. }\end{array}$ & Age & Sex & Karyotype & Clinical feature \\
\hline 52 & $37 \mathrm{y}$. & $\mathrm{F}$ & $46, \mathrm{XX}$ & $\begin{array}{l}\text { short stature, low birth weight, hirsutism, microcephaly, } \\
\text { short neck. teeth anomaly and irregular placement. rocker } \\
\text { bottom. palsy of extremity, hypertonicity, ataxia, disturb- } \\
\text { ance of gaits, idiot }\end{array}$ \\
\hline 53 & $28 \mathrm{y}$. & $\mathrm{F}$ & $46, \mathrm{XX}$ & $\begin{array}{l}\text { short neck, protrusion of labium inferius, ataxia, disturb- } \\
\text { ance of gaits, idiot }\end{array}$ \\
\hline 54 & $28 \mathrm{y}$. & $\mathrm{F}$ & $46, \mathrm{XX}$ & $\begin{array}{l}\text { brachycephaly, short neck, facial telangiectatic erythema, } \\
\text { round face. saddle nose, athetotic movement, ataxia, dis- } \\
\text { turbance of gaits, idiot }\end{array}$ \\
\hline 55 & $11 \mathrm{y}$. & $\mathrm{F}$ & $46, \mathrm{XX}$ & $\begin{array}{l}\text { mask-like facies, hypertelorism, upward slanted palpebral } \\
\text { fissure, saddle nose, protrus:on of labium inferius, disloca- } \\
\text { tion of hip, hypotonicity, ataxia, disturbance of gaits, idiot }\end{array}$ \\
\hline $56 *$ & $8 \mathrm{y}$. & F & $46, \mathrm{XX} / 46, \mathrm{XX}$ & $\begin{array}{l}\text { r(9)(p24q34) } \\
\text { slow growth, malnutrition, short stature, dry skin, hir- } \\
\text { sutism, microcephaly, trigonocephaly, long eyelashes, } \\
\text { slight down-ward slanted palpebral fissure, mask-like } \\
\text { facies, macroglossia, protruding tongue, malformed low } \\
\text { set ear, teeth anomaly and irregular placement, webbed } \\
\text { neck, wide-spaced nipple, joint hyperextensibility, palsy } \\
\text { of extremity, reflex acceleration, arachnodactyly, repeated } \\
\text { convulsion, ataxia, disturbance of gaits, idiot }\end{array}$ \\
\hline 57 & $22 \mathrm{y}$. & F & $46, \mathrm{XX}$ & $\begin{array}{l}\text { slow growth, malnutrition, short stature, skin pigmenta- } \\
\text { tion, nevus vasculosus, dry skin, flat occiput, funnel breast, } \\
\text { incomplete development of external genitalia, ataxia, idiot }\end{array}$ \\
\hline 58 & $39 \mathrm{y}$. & F & $46, \mathrm{XX}$ & $\begin{array}{l}\text { slow growth, malnutrition, short neck, facial telangiectatic } \\
\text { erythema, hypertelorism, saddle nose, low nasal bridge, } \\
\text { protrusion of labium inferius, brachydactyly, teeth anoma- } \\
\text { ly and irregular placement, V. finger clinodactyly, IV toe } \\
\text { hypoplasia, ataxia, disturbance of gaits, idiot }\end{array}$ \\
\hline $59 *$ & $25 \mathrm{y}$. & $\mathrm{M}$ & $46, \mathrm{XYq}-$ & $\begin{array}{l}\text { clubhand, clubfoot, equinus, palsy of extremity, hyper- } \\
\text { tonicity, ataxia, disturbance of gaits, imbecile }\end{array}$ \\
\hline 60 & $22 \mathrm{y}$. & $\mathrm{F}$ & $46, \mathrm{XX}$ & facial telangiectatic erythema, idiot \\
\hline 61 & $25 \mathrm{y}$. & $\mathrm{M}$ & $46, \mathrm{XY}$ & $\begin{array}{l}\text { dolichocephalia, flat occiput, facial telangiectatic ery- } \\
\text { thema, cataract (right), incomplete development of exter- } \\
\text { nal genitalia, vagus, ataxia, disturbance of gaits, idiot }\end{array}$ \\
\hline 62 & $27 \mathrm{y}$. & M & $46, \mathrm{XY}$ & slow growth, ataxia, idiot \\
\hline $63 *$ & $18 \mathrm{y}$. & M & $46, X Y, 21 p--$ & $\begin{array}{l}\text { hirsutism, recurrent cramp, ataxia, disturbance of gaits, } \\
\text { idiot }\end{array}$ \\
\hline 64 & $28 \mathrm{y}$. & $\mathrm{F}$ & $46, \mathrm{XX}$ & short neck, ataxia, disturbance of gaits, idiot \\
\hline 65 & $11 \mathrm{y}$. & $\mathrm{M}$ & $46, \mathrm{XY}$ & $\begin{array}{l}\text { dry skin, hirsutism, flat occiput, strabismus (left), weak } \\
\text { sight, recurrent cramp, ataxia, disturbance of gaits }\end{array}$ \\
\hline 66 & $36 \mathrm{y}$. & M & $46, \mathrm{XY}$ & $\begin{array}{l}\text { slow growth, malnutrition, short stature, small cranium, } \\
\text { older facies, prominent ears, clubhand, clubfoot, cavus, } \\
\text { ataxia, disturbance of gaits, idiot }\end{array}$ \\
\hline 67 & $37 \mathrm{y}$. & M & $46, \mathrm{XY}$ & $\begin{array}{l}\text { skin pigmentation, dry skin, short neck, round face, exoph- } \\
\text { thalmus, macroglossia, wide cleft between I. and II. toe, } \\
\text { idiot }\end{array}$ \\
\hline $68^{*}$ & $11 \mathrm{y}$. & M & $47, \mathrm{XY},+21$ & $\begin{array}{l}\text { slow growth, malnutrition, short stature, microcephaly, } \\
\text { hypertelorism, strabismus, blepharophimosis, blepharitis, } \\
\text { saddle nose, macroglossia, joint hyperextensibility, simian- } \\
\text { line, ataxia, disturbance of gaits, idiot }\end{array}$ \\
\hline 69 & $22 \mathrm{y}$. & M & $46, \mathrm{XY}$ & $\begin{array}{l}\text { albino, flat occiput, red hair, round face, protrusion of } \\
\text { labium inferius, idiot }\end{array}$ \\
\hline 70 & $15 \mathrm{y}$ & $\mathrm{F}$ & $46, \mathrm{XX}$ & $\begin{array}{l}\text { round face, saddle nose, low nasal bridge, teeth anomaly } \\
\text { and irregular placement. thick lip, female like breast, re- } \\
\text { current cramp, ataxia, disturbance of gaits, imbecile }\end{array}$ \\
\hline 71 & $18 \mathrm{y}$. & M & $46, \mathrm{XY}$ & $\begin{array}{l}\text { flat occiput, protrusion of labium inferius, teeth anomaly } \\
\text { and irregular placement, hypodontia, pigeon breast, ataxia, } \\
\text { disturbance of gaits, idiot }\end{array}$ \\
\hline 72 & $14 \mathrm{y}$. & M & $46, \mathrm{XY}$ & weak sight, idiot \\
\hline 73 & $34 \mathrm{y}$ & M & $46, \mathrm{XY}$ & $\begin{array}{l}\text { dolichocephalia, flat occiput, aged facies, inner epicanthal } \\
\text { fold, malformed auricle, prognathism. narrow high arched } \\
\text { palate, ataxia, disturbance of gaits, idiot }\end{array}$ \\
\hline $74 *$ & $30 \mathrm{y}$. & M & $46, \mathrm{XYq}-$ & low birth weight, flat occiput, idiot \\
\hline 75 & $18 \mathrm{y}$. & $\mathrm{F}$ & $46, \mathrm{XX}$ & $\begin{array}{l}\text { low birth weight, dry skin, microcephaly, flat occiput, } \\
\text { protrusion of labium inferius, teeth anomaly and irregular } \\
\text { placement, palsy of extremity, recurrent cramp, disturb- } \\
\text { ance of gaits, idiot }\end{array}$ \\
\hline
\end{tabular}


Table I (Continued)

\begin{tabular}{|c|c|c|c|c|}
\hline $\begin{array}{l}\text { Case } \\
\text { no. }\end{array}$ & Age & Sex & Karyotype & Clinical feature \\
\hline 76 & $14 \mathrm{y}$. & $\mathrm{M}^{\prime}$ & $46, \mathrm{XY}$ & short neck, round face, weak sight, idiot \\
\hline $77 *$ & $11 \mathrm{y}$. & M & $46, \mathrm{XYq}-$ & $\begin{array}{l}\text { slow growth, teeth anomaly and irregular placement, thick } \\
\text { lip, left hemiplegie, palsy of extremity, muscle atrophy, } \\
\text { recurrent cramp, ataxia, disturbance of gaits, idiot }\end{array}$ \\
\hline $78 *$ & $36 \mathrm{y}$. & $\mathrm{F}$ & $46, \mathrm{XX}, 21 \mathrm{~s}+$ & $\begin{array}{l}\text { dry skin, exophthalmus, malformed auricle, malformed } \\
\text { limbs, recurrent cramp, ataxia, idiot }\end{array}$ \\
\hline $79 *$ & $16 \mathrm{y}$. & $\mathrm{M}$ & $46, X Y, 15 p+$ & $\begin{array}{l}\text { short stature, macrocephaly, short neck, round face, ptosis } \\
\text { of eyelid, small nose, teeth anomaly and irregular place- } \\
\text { ment, short and sparse cilia, incomplete development of } \\
\text { internal and external genitalia, small penis, short extre- } \\
\text { mities, ataxia, disturbance of gaits, idiot }\end{array}$ \\
\hline $80 *$ & $30 \mathrm{y}$. & $\mathrm{M}$ & $46, \mathrm{XY}, 14 \mathrm{~s}+$ & $\begin{array}{l}\text { skin pigmentation, exophthalmus, protrusion of labium in- } \\
\text { ferius, teeth anomaly and irregular placement, idiot }\end{array}$ \\
\hline 81 & $28 \mathrm{y}$. & $\mathrm{M}$ & $46, \mathrm{XY}$ & cataract, blindness, idiot \\
\hline 82 & $19 \mathrm{y}$. & M & $46, \mathrm{XY}$ & varus, simianline, debility \\
\hline 83 & $27 \mathrm{y}$. & $\mathrm{M}$ & $46, \mathrm{XY}$ & $\begin{array}{l}\text { hirsutism, microcephaly, flat occiput, blepharitis, blind- } \\
\text { ness, prominent nasal bridge, teeth anomaly and irregular } \\
\text { placement, ataxia, disturbance of gaits, idiot }\end{array}$ \\
\hline 84 & $9 \mathrm{y}$. & M & $46, \mathrm{XY}$ & $\begin{array}{l}\text { microcephaly, brachycephaly, round face, protrusion of } \\
\text { labium inferius, rocker bottom, palsy of extremity, ataxia, } \\
\text { disturbance of gaits, idiot }\end{array}$ \\
\hline 85 & $27 \mathrm{y}$. & $\mathrm{F}$ & $46, \mathrm{XX}$ & $\begin{array}{l}\text { dry skin, blepharophimosis, nose depressed, long II. toe, } \\
\text { recurrent cramp, ataxia, disturbance of gaits, idiot }\end{array}$ \\
\hline $86 *$ & $12 \mathrm{y}$. & $\mathrm{M}$ & 46,XY,der(1), & $\begin{array}{l}\text { ins }(1 ; 9)(\mathrm{q} 11 ; \mathrm{q} 12 \mathrm{q} 13) \text { mat } \\
\text { goose flesh, dry and hard skin, dolichocephalia, flat occi- }- \text { har } \\
\text { put, upward slanted palpebral fissure, blepharophimosis, } \\
\text { weak sight, nose depressed, low nasal bridge, teeth anoma- } \\
\text { ly and irregular placement, low set ear, incomplete de- } \\
\text { velopment of internal and external genitalia, cryptor- } \\
\text { chism, small penis, clubfoot, varus, repeated convulsion, } \\
\text { imbecile }\end{array}$ \\
\hline 87 & $34 \mathrm{y}$. & $\mathrm{M}$ & $46, \mathrm{XY}$ & father of the case no. 86 \\
\hline $88 *$ & $30 \mathrm{y}$. & $\mathrm{F}$ & $46, \mathrm{XX}, \mathrm{ins}(1 ; 9$ & $\begin{array}{l}\text { 9)(q11; } 12 q 13) \\
\text { mother of the case no. } 86 \text {, normal phenotype }\end{array}$ \\
\hline 89 & $29 \mathrm{y}$. & $\mathrm{F}$ & $46, \mathrm{XX}$ & hirsutism, round face, idiot \\
\hline 90 & $24 \mathrm{y}$. & M & $46, \mathrm{XY}$ & $\begin{array}{l}\text { hirsutism, upward slanted palpebral fissure, saddle nose, } \\
\text { macroglossia, clubfoot, palsy of leg, ataxia, disturbance of } \\
\text { gaits, idiot }\end{array}$ \\
\hline $91^{*}$ & $11 \mathrm{y}$. & M & $46, \mathrm{XYq}+$ & $\begin{array}{l}\text { low birth weight, dolichocephalia, dry skin, facial telangiec- } \\
\text { tatic erythema, nose depressed, incomplete development of } \\
\text { internal and external genitalia, short limbs, brachydacty- } \\
\text { ly, debility }\end{array}$ \\
\hline 92 & $12 \mathrm{y}$. & $\mathrm{F}$ & $46, \mathrm{XX}$ & idiot \\
\hline
\end{tabular}

Cases with asterisks are chromosomally abnormal.

normal long arm of no. 3 resulting the partial duplication of the long arm of no. $3(q 22 \rightarrow q 27)(46, X X$, dir dup (3) $(q 22 \rightarrow q 27))$; one case of a partial trisomy for the long arm of no. $9(46, X Y$,der (1),ins $(1 ; 9)$ (q11;q12q13) mat); and one case showing a direct insertion of the long arm of no. 9 into the long arm of no. $1(46, X X$, ins $(1 ; 9)$ (q11; q12q13) ).

Among the abnormalities, one case of the partial duplication of the long arm of no. 3 (case no. 16) seemed to be of sporadic origin, since the parents of this case were chromosomally normal.*) Details of the case with a mosaicism with ring no. 9 (case no. 56) were reported by Kadotani et al.**) Partial trisomy for the long arm of no. 9

*) Kadotani et al.: Proc. Japan Acad., vol. 55B, pp. 431-435, 1979.

**) Kadotani et al.: Proc. Japan Acad., vol. 56B, pp. 85-88, 1980. 
[Vol. $56(\mathrm{~B})$,

(case no. 86) was transmitted through the maternal line, since the mother of this case (case no. 88) was a carrier of the direct insertion of a long arm of no. 9 into a long arm of no. 1.*)

Acknowledgements. We are much obliged to Emeritus Professor Sajiro Makino, M. J. A., the senior consultant of the Kadotani Medical Research Foundation, for improvement of this manuscript, and to Mrs. Keiko Sasaki for technical assistance. Financial aid from the Japan Academy is gratefully acknowledged here.

*) Kadotani et al.: Proc. Japan Acad., vol. 56B, pp. 157-161, 1980. 